\title{
Multiple wavelength optical observations of a long-lived meteor trail
}

\author{
B. R. Clemesha, A. F. de Medeiros, D. Gobbi, H. Takahashi and P. P. Batista \\ Instituto Nacional de Pesquisas Espaciais, 12201-970, S. J. dos Campos, SP, Brazil
}

\author{
M. J Taylor \\ Space Dynamics Laboratory and Physics Department, Utah State University, Logan, Utah, USA
}

\begin{abstract}
A long-lived meteor trail has been observed at wavelengths of $572.5 \mathrm{~nm}, 557.7 \mathrm{~nm}, 630.0 \mathrm{~nm}, 865.5 \mathrm{~nm}$ and in the near infrared band from 715 to $930 \mathrm{~nm}$. The trail was detected at all these wavelengths, with the possible exception of $865.5 \mathrm{~nm}$, where its identification was marginal. It was seen longest (17 minutes) through the wide band NIR 715 $930 \mathrm{~nm}$ filter. The fact that the trail was only marginally visible in the $865.5 \mathrm{~nm}(0-1)$ band of molecular oxygen, and was strongest in the wide-band NIR image, raises serious doubts about an earlier suggestion that the infrared light from longlived meteor trails corresponds to emissions from molecular oxygen excited by the Chapman mechanism.
\end{abstract}

\section{Introduction}

For many years there have been occasional reports in the literature of meteor trails which persist for up to one hour. Thess observations are usually associated with particularly strong meteor showers, such as the Leonids. In a recent paper, Kelley et al. [2000] have presented some very interesting observations of such trails associated with the 1998 Leonids event. In their paper Kelley et al. also reproduced an illustration from a paper by Trowbridge [1907] who reported early visual observations made during the 1866 Leonids. The interest in this phenomenon lies, of course, in the mechanism which can cause a meteor trail to emit light for as much as 60 min. The measurements of Kelley et al. [2000] went beyond anything previously published in that, in addition to obtaining images of the trails with the aid of intensified CCD cameras, they were also able to track the trails by means of a sodium lidar. By means of such measurements they were able to show that the Chapman [1939] mechanism, generally accepted as responsible for the night airglow in the $\mathrm{NaD}$ lines, and suggested by Chapman [1956] as a candidate for long-lived meteor trail glow, would not be strong enough to explain the total light intensity seen by a wide-band CCD camera. In this they confirm the earlier work of Baggaley [1975]. The purpose of the present paper is to report the observation of a long-lived meteor trail at 4 different wavelengths in the visible and near infrared. Although these new observations do not enable us to identify the mechanism involved, they do allow us to reevaluate one of the mechanisms suggested in earlier work.

\section{Copyright 2001 by the American Geophysical Union.}

\section{Observations}

The observations were made using a Utah State University, monochromatic all-sky CCD imager designed for observing the airglow emissions [Taylor et al., 1997]. The camera has been operating at Cachoeira Paulista, Brazil $\left(23^{\circ} \mathrm{S}, 45^{\circ} \mathrm{W}\right)$ since the Austral Spring 1998 as part of a collaborative program with INPE. The imager is fitted with a filter wheel with filters for the OI $577.7 \mathrm{~nm}$ and $630 \mathrm{~nm}$ atomic oxygen emissions, the $\mathrm{O}_{2}(0-1)$ band at $865.5 \mathrm{~nm}$, a wide band near infrared (NIR) filter, passing 715 to $930 \mathrm{~nm}$, and a background (BG) filter centered on $572.5 \mathrm{~nm}$. The NIR filter, which passes the $8-3 ; 4-0 ; 9-4 ; 5-1 ; 6-2 ; 7-3$ and part of 8-4 Meinel bands, has a notch at $865.5 \mathrm{~nm}$ so that it strongly rejects the molecular oxygen emission. The characteristics of the imager are summarized in Table 1 . The images shown in this paper cover a roughly $60^{\circ}$ wide section of the sky centered near Right Ascension 0, Declination $-36^{\circ}$.

In Figure 1 we show three images obtained through the $557.7 \mathrm{~nm}$ filter at intervals of about $3.5 \mathrm{~min}$ on July 16, 1999. The first image, at 05:17:17 UT shows a bright meteor trail terminating in a fireball located at a Right Ascension of $7 \mathrm{~min}$ and Declination $36^{\circ}$. Three and a half minutes later a very faint, but typically wind-distorted, luminous trail is still visible. In the last image, just over 7 minutes after the initial fireball, a distorted trail is still just visible. Much clearer images of the meteor trail can be seen in Figure 2, where we show the images obtained through the NIR filter. The first NIR image, obiained just over 3 minutes after the initial fireball, shows a bright trail, already showing the effects of wind-distortion. The following 4 images show decreasing intensity and further wind-distortion. The last image on which the trail is still visible was obtained about 17 minutes after the meteor event. Note that the NIR sequence shows the normal $\mathrm{OH}$ airglow emission modulated by a gravity wave propagating towards the SW. In Figure 3 we show 2 images taken through the 630 $\mathrm{nm}$ filter. The first image, taken about one and a half minutes

Table 1. Imager characteristics

\begin{tabular}{llll}
\hline Emission / Filter & $\begin{array}{l}\text { Exposure } \\
\text { Time }(\mathrm{s})\end{array}$ & $\begin{array}{l}\text { Transmission } \\
(\%)\end{array}$ & $\begin{array}{l}\text { Bandwidth } \\
(\mathrm{nm})\end{array}$ \\
\hline NIR OH & 15 & 87 & $715-930^{*}$ \\
BG $(572.5 \mathrm{~nm})$ & 90 & 83 & 2.67 \\
$\left.\mathrm{O}_{2} \dot{\mathrm{i}}(\mathrm{i})-1\right)(865.5 \mathrm{~nm})$ & 90 & 84 & 12.0 \\
OI $(557.7 \mathrm{~nm})$ & 90 & 81 & 2.65 \\
OI $(630.0 \mathrm{~nm})$ & 90 & 83 & 3.30 \\
\hline
\end{tabular}

"With a $20.0 \mathrm{~nm}$ notch at $865.5 \mathrm{~nm}$. 

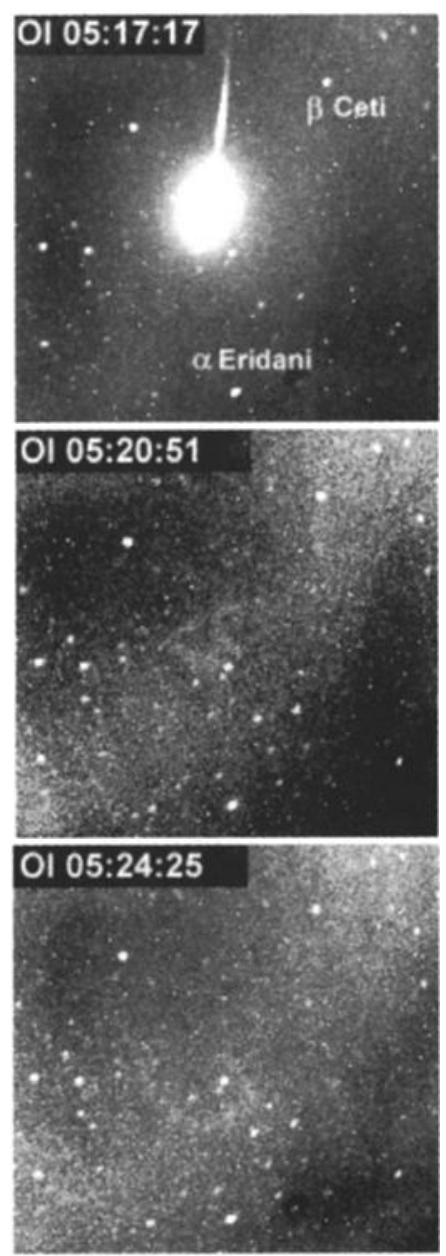

Figure 1. Sequence of images taken through a $557.7 \mathrm{~nm}$ filter. Times are given in UT.

after the initial fireball, shows a short trail, pointing in the direction of the original meteor trn $^{\circ} \mathrm{k}$ visible in Figure 1, and a curiously diffuse blob, in approximately the position of the original fireball. The second image, taken nearly 9 minutes later, shows a very faint trail with the shape and position expected from the time sequence of much stronger NIR images shown in Figure 2. The last figure shows the images obtained through the $865.5 \mathrm{~nm}$ and $572.5 \mathrm{~nm}$ filters. The $865.5 \mathrm{~nm}$ image was taken immediately after the second of the NIR images and possibly shows a very faint trail in the same position as that seen in the NIR image. We show this image in order to demonstrate that any emission in the (0-1) band of molecular oxygen was extremely weak. The feature marked "simulated trail" in this image will be discussed later. The second of the images of Figure 4, taken through the $572.5 \mathrm{~nm}$ filter just over 12 minutes after the initial observation, shows a faint trail in the position expected from the NIR sequence.

\section{Discussion}

To explain long-lived meteor trails observed in NIR airglow imagery Hapgood [1980] suggested that the trails could involve emissions from the atmospheric band of molecular oxygen excited by the Chapman mechanism, generally accepted for the sodium nightglow, and probably responsible for a major part of the emission observed from meteor trails at visible wavelengths [Chapman, 1939, 1956]. Hapgood pointed out that with an exothermicity of $2.5 \mathrm{eV}$ there are 4 variations of the basic reaction that are energetically and spin allowed:
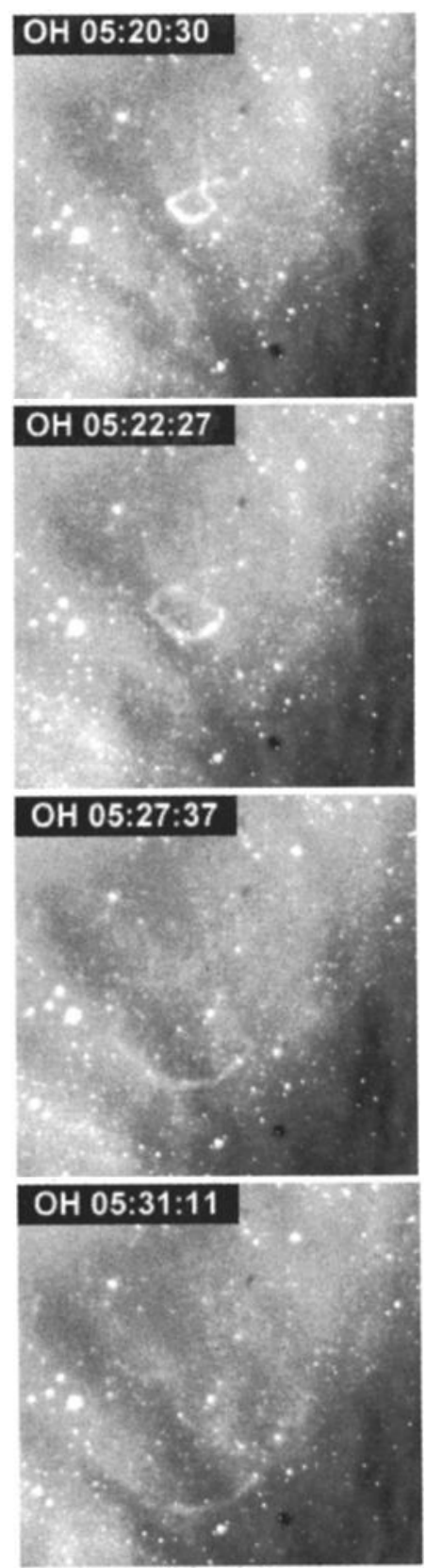

OH 05:34:44

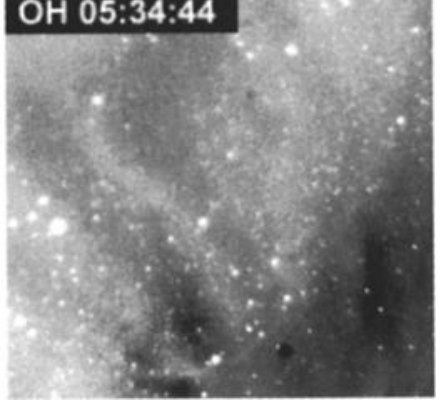

Figure 2. As for Figure 1, but for wide-band NIR filter. 

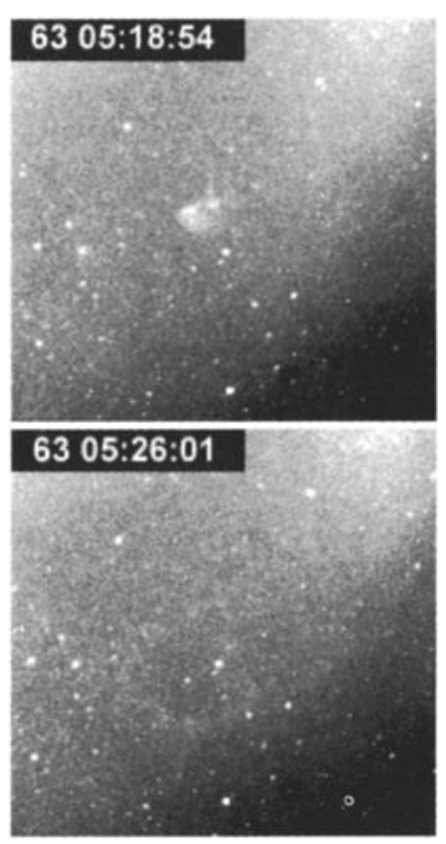

Figure 3. As for Figure 1, but for $630 \mathrm{~nm}$ filter.

$$
\begin{array}{rll}
\mathrm{NaO}\left(\mathrm{X}^{2} \pi\right)+\mathrm{O}\left({ }^{3} \mathrm{P}\right) \rightarrow & \mathrm{Na}\left({ }^{2} \mathrm{~S}\right)+\mathrm{O}_{2}\left(\mathrm{X}^{3} \Sigma\right) & 0 \mathrm{eV} \\
& \mathrm{Na}\left({ }^{2} \mathrm{~S}\right)+\mathrm{O}_{2}\left(\mathrm{a}^{1} \Delta\right) & 0.98 \mathrm{eV} \\
& \mathrm{Na}\left({ }^{2} \mathrm{~S}\right)+\mathrm{O}_{2}\left(\mathrm{~b}^{1} \Sigma\right) & 1.63 \mathrm{eV} \\
& \mathrm{Na}\left({ }^{2} \mathrm{P}\right)+\mathrm{O}_{2}\left(\mathrm{X}^{3} \Sigma\right) & 2.09 \mathrm{eV}
\end{array}
$$

where the energies shown are those necessary to excite the lowest vibrational state in the upper electronic level. It is estimated that branch $\mathrm{d}$ of this reaction, responsible for the $\mathrm{Na}$ nightglow, represents only about $10 \%$ of the total [Clemesha et il., 1995], so a significant fraction could go in the direction of branch $\mathrm{c}$, leading to the production of $\mathrm{O}_{2}\left(\mathrm{~b}^{\prime} \Sigma\right)$. Kelley $e t$ al. [2000] discussed this possibility and pointed out that, although they saw long-lived trails with a CCD camera designed for imaging the NIR OH bands, they could not exclude the possibility that the main emission might have been from molecular oxygen, because their NIR filter included the $\mathrm{O}_{2}(0-$ 1) band. In the present work, not only did our NIR filter have a notch at $865.5 \mathrm{~nm}$, but we also have an image taken at this wavelength. The fact that we see a strong trail with the NIR filter, and an only marginally visible one through the 865.5 $\mathrm{nm}$ filter, makes it quite certain that the main infrared emission cannot be from the (0-1) band of molecular oxygen.

Hapgood [1980] pointed out that the Chapman mechanism is sufficiently exothermic to excite $\mathrm{O}_{2}\left(b^{1} \Sigma\right)$ to vibrational levels up to $v=5$, and could thus lead to emissions at many wavelengths within the passband of his camera. Our present data show that there is negligible emission in the (0-1) band, but does not eliminate the possibility of emissions from other vibrational transitions in the NIR. Only recently have bands other than the (0-1) and (0-0) been detected in the terrestrial airglow (note that the (0-0) band is strongly absorbed in the lower atmosphere and thus is not visible from the ground). Slanger et al. [2000] have observed vibrational levels up to 15 in data from the HIRES spectrometer on the Keck telescope. These workers find a total intensity of about $150 \mathrm{R}$ for bands with $v^{\prime}=1-15$. Since the (0-1) airglow intensity is typically about $300 \mathrm{R}$, and the transition probabilities for $\Delta \mathrm{v}>1$ are much less than for $\Delta v=1$, about $2 / 3$ of the $\mathrm{O}_{2}$ atmospheric band intensity visible from the ground should be in the (0-1) emission. Bands corresponding to higher vibrational levels have also been observed in the aurora, and Vallance Jones [1974] gives estimates for the relative intensity of bands up to $v^{\prime}=7, v^{\prime \prime}=9$ in his Table 4.15. Summing over all these transitions, excluding the $(0-0)$ band, we find that approximately $50 \%$ of the remaining intensity should be in the (0-1) transition. We conclude that the auroral and nightglow observations indicate that the intensity of the $(0-1)$ band should be at least as great as that of all the other bands together, with the exception of the (0-0) band, not visible from the ground. Since the Chapman mechanism can produce $\mathrm{O}_{2}\left(b^{i} \Sigma\right)$ in vibrational levels only up to $v=5$ (or $v=6$ according to more recent work [Joo et al., 1999]), there is no reason to believe that the atmospheric band emission that might result from this mechanism should have a higher fraction of its total intensity in transitions involving high vibrational levels. These considerations suggest that it is extremely unlikely that emissions in the $\mathrm{O}_{2}$ Atmospheric bands excited by the Chapman mechanism could have produced the NIR glow observed by our camera.

The fact that we saw much the strongest images through the NIR filter suggests that the main emission was from the $\mathrm{OH}$ bands. On the other hand, if this is the case the images seen at $557.7,630.0$ and $572.5 \mathrm{~nm}$ are unexpectedly strong. The $\mathrm{P}$ branch of the $\mathrm{OH}(7-1)$ band contains lines which fall within the pass-band of the $557.7 \mathrm{~nm}$ filter, the $630 \mathrm{~nm}$ filter should pass lines from the $\mathrm{OH}$ (9-3) $\mathrm{P}$ branch, and lines corresponding to rotational levels greater than 6 in the $P$ branch of the $\mathrm{OH}(7-1)$ band would pass the $572.5 \mathrm{~nm}$ background filter. In the night airglow, all these components of the $\mathrm{OH}$ emission are extremely weak and usually undetectable. Excitation in the meteor trail could well be different to that responsible for the airglow, so the fact that emissions were seen at the "non$\mathrm{OH}^{\prime \prime}$ wavelengths does not necessarily indicate the existence of emissions other than $\mathrm{OH}$. On the same basis, of course, we cannot entirely rule out the possibility of an unknown mecha-
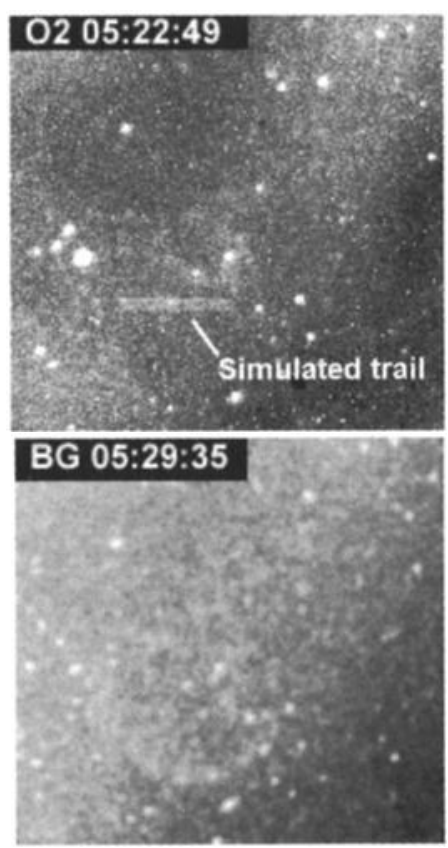

Figure 4. As for Figure 1, but for $865.5 \mathrm{~nm}$ (top panel) and $572.5 \mathrm{~nm}$ (bottom panel) respectively. 
nism that produces a vibrational distribution in the $\mathrm{O}_{2}$ Atmospheric band quite different to that seen in the airglow and aurora.

Another possibility that wouid explain the much stronger trail seen through the wide-band NIR filter is that the main emission is a continuum. To investigate this possibility we have made a rough calculation of the expected $865.5 \mathrm{~nm}$ signal, assuming a uniform spectral intensity over the observed Meinel bands and taking into account the different bandwidths and exposure times. Since the sensitivity of the CCD device does not vary greatly over the wavelength range involved we have neglected this factor. The result is shown in Figure 4 as a simulated trail. It is clear that the observed $865.5 \mathrm{~nm}$ signal is less than that which would be seen in the case of a uniform continuum, indicating at the very least that the assumption of constant spectral intensity is not valid, and thus providing evidence against the continuum possibility.

An interesting feature of the trails seen by other workers is the bright edge and dark interior (see for example, Plate 1 of Kelley et al., 2000). Our images have insufficient spatial resolution to show this effect - the trails are only a few pixels wide. Kelley et al. [2000] suggested that the effect could occur if the glow is from Na excited by the Chapman mechanism and ozone is depleted inside the trail, and Zinn et al. [1999] suggested that the ozone could be destroyed by photolysis during the initial fireball stage of the trail. It seems to us that these speculations are unnecessarily restrictive. Any process involving reactions between meteoric material and atmospheric constituents, most probably odd oxygen or hydrogen, could lead to this effect. The atmospheric constituents would be highly depleted inside the meteor trail, and their replenishment would occur via molecular diffusion. This would inevitable lead to the glow-producing reactions occurring only on the boundaries of the trail. Thus the observation of a dark interior to the trail makes it almost certain that the long-duration glow is produced by reactions between meteoric material and atmospheric constituents, rather than by a process which involves only the meteor material.

A tinal point of interest is the diffuse emission visible in the first $630 \mathrm{~nm}$ image taken about 90 seconds after the initial fireball. This emission region is wider than the trail seen in any of the other images, and must thus exceed the boundaries of the region in which meteor ablation products are deposited. This could be a remnant of the initial fireball, but we might also speculate that the glow results from the recombination of atmospheric constituents dissociated by the initial intense UV radiation from the fireball.
Acknowledgments. We gratefully acknowledge the financial support of the Programa de Núcleos de Excelência - Pronex, the Fundação de Amparo a Pesquisa do Estado de São Paulo - FAPESP and the Conselho Nacional de Desenvolvimento Científico e Tecnológico - CNPq. The USU camera and operations were supported by NSF grant No ATM-9525815.

\section{References}

Baggaley, W. J., Sodium Emission From Long Enduring Meteor Truins Nature 257: 567-568 1975

Chapman, S., Notes on atmospheric sodium, Astrophys. J., 90, 309$316,1939$.

Chapman, S., Note on persistent meteor trails, in The Airglow and the Aurorae. A. Delgano and E. B. Armstrong, eds., 204-205, 1956.

Clemesha, B. R., D. M. Simonich, H. Takahashi, S. M. L. Melo and J. M. C. Plane, Experimental evidence for photochemical control of the atmospheric sodium layer, J. Geophys. Res., 100, 18,909i: $: 916,1995$

Hapgood, M. A., IR observation of a persistent meteor train, Nature, $286,582-583,1980$.

Joo, S , D. R. Worsnop, C. E. Kolb, S. K. Kim and D. R. Herschbach, Observation of the A-X electronic transition of Na, J. Phys. Chem. A, 103, 3193-3199, 1999.

Kelley, M. C., C. S. Gardner, J. Drummond, T. Armstrong, A. Liu, X. Clu, G. Papen, C. Kruschwitz, P. Loughmiller, B. Grime, et al., First observations of long-lived meteor trains with resonance lidar and other optical instruments, Geophys. Res. Lett., 27, 1811-1814, 2000.

Slanger, T. G., P. C. Cosby, D. L. Huestis and D. E. Osterbrock, Vibrational level distribution of $\mathrm{O}_{2}\left(\mathrm{~h}^{1} \Sigma^{+}, \mathrm{i}^{\prime}=0-15\right)$ in the mesosphere and lower thermosphere region, J. Geophys. Res., 105, 20557-20564, 2000.

Taylor, M. J. Pendleton Jr., W. R. Clark, S. Takahashi, H. Gobbi, D. Goldberg, R. A. Image measurements of short-period gravity waves at equatorial latitudes, J. Geophys. Res., 102, 26283-26299, 1997.

Trowbridge, C. C., The origin of luminous meteor trains, Popular Science Monthly, August, 402-407, 1907.

Vallance Jones, A., Aurora, Geophystcs and Astrophysics Monographs, 9, 136, 1974.

Zinn, J., J. Wren, R. Whitaker, J. Szymanski, D. O. Revelle, W. $P_{1}$ iedhorsky, J. Hills, G. Gisler, S. Fletcher, D. Casperson, et al., (uordinated observations of two large Leionid meteor fireballs over northern New Mexico and computer comparisons, Meteor. Planet. Sc1., 34, 1007-1016, 1999.

B.R. Clemesha, A. F. Medeiros, D. Gobbi, H. Takahashi and P. P. Batsta, Instituto Nacional de Pesquisas Espaciais, CP 515, S. J. dos Campos, 12201-970 SP, Brazil. (clem@laser.inpe.br; afragoso@laser.inpe.br; delano@laser.inpe.br; hisao@laser.inpe.br; pbatista@laser.inpe.br)

M. J Taylor, Space Dynamics Laboratory and Physics Department, Utah State University, Logan, Utah, USA (mtaylor@cc.usu.edu)

(Received: November 9, 2000; Revised: F.L.uary 9, 200)! Accepted: May 13, 2001) 